This document is confidential and is proprietary to the American Chemical Society and its authors. Do not copy or disclose without written permission. If you have received this item in error, notify the sender and delete all copies.

\title{
Mechanical Stabilization of Helical Chirality in a Macrocyclic Oligothiophene
}

\begin{tabular}{|r|l|}
\hline Journal: & Journal of the American Chemical Society \\
\hline Manuscript ID & ja-2018-11797k.R2 \\
\hline Manuscript Type: & Article \\
\hline Complete List of Authors: & $\begin{array}{l}\text { Weiland, Kevin; University of Basel, } \\
\text { Brandl, Thomas; University of Basel, Department of Chemistry } \\
\text { Atz, Kenneth; University of Basel, Department of Chemistry } \\
\text { Prescimone, Alessandro; Universitat Basel, Chemistry } \\
\text { Häussinger, Daniel; Universitat Basel, Chemie } \\
\text { Solomek, Tomas; University of Basel, Department of Chemistry } \\
\text { Mayor, Marcel; Universitat Basel, Department of Chemistry; Karlsruhe } \\
\text { Institute of Technology (KIT), Institute for Nanotechnology (INT); Sun } \\
\text { Yat-Sen University, Lehn Institute of Functional Materials }\end{array}$ \\
\hline \multicolumn{2}{|l}{} \\
\hline
\end{tabular}

\section{SCHOLARONE Manuscripts}




\section{INTRODUCTION}

Helically chiral aromatic molecules, ${ }^{1-3}$ besides their esthetic beauty, often possess interesting chiroptical properties such as intense circular dichroism or spin-selective electron transport. ${ }^{4-6}$ Apart from ubiquitous helicenes, [2.2] paracyclophane (PC) ${ }^{7,8}$ represents an interesting building block to form helical structures because tetrasubstituted PCs, prepared initially by Hopf and co-workers, are chiral. For instance, Chujo and coworkers demonstrated the synthesis of enantiopure doubly-helical macrocycles and discovered their intense chiroptical effects, ${ }^{9}, 10$ which were concluded to arise from the rigid geometry of the propeller-shaped molecules.

Our interest in "loop-shaped" helically chiral model compounds arises from their potential physical properties in single-molecule junctions. To investigate the suitability of the PC synthon, an achiral PC derivative was recently analyzed in a single-molecule junction displaying mechanically triggered conductance variations. ${ }^{11}$ As the next step, we decided to investigate the PC building block as a helicity-inducing subunit in macrocycle $\mathbf{2 b}$ (figure 1 ). We found the helically chiral molecule to enantiomerize rapidly in solution and therefore decided to stabilize the helical chirality by mechanically hindering the racemization through strategic installation of bulky substituents. $^{12}$

Disconnection of a $\mathrm{C}-\mathrm{C}$ bond between two thiophenes in a planar oligothiophene macrocycle (figure $1 \mathrm{~b}$ ) and attaching each of the termini to the pseudo-para-position of the top and the bottom benzene ring in PC, respectively, introduces a step in the macrocyclic ring, from which the helicity of the resulting PC macrocycle emanates (figure $1 \mathrm{c}, \mathrm{d}$ ). The oligothiophene macrocycle $\mathbf{2 b}$ (figure 1d) com- a)

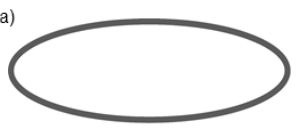

c)

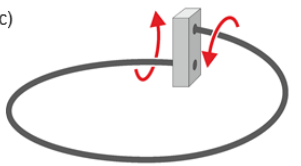

e)

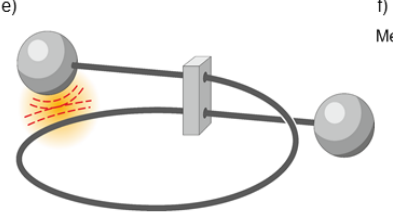

b)

d)
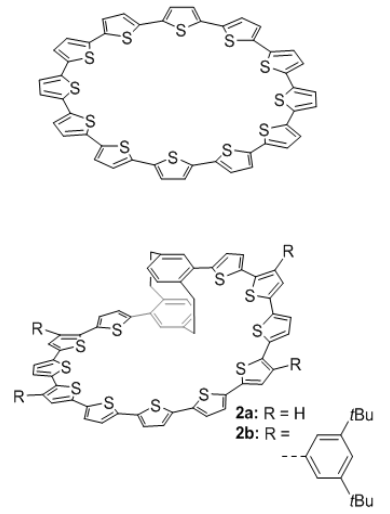

Figure 1. Development of the design of the mechanically stabilized helical chiral PC-macrocycle 1. Starting from the planar ring (a) represented by cyclo[12] thiophene (b), the introduction of a step results in a helical chiral object (c) as in the oligothiophene macrocycle 2 comprising a PC subunit (d). Additional decoration of the PC subunit with bulky substituents hinders its enantiomerization by rotation of the PC subunit as sketched in (e). The concept is realized with the macrocyclic model compound 1 reported here.

prising a pseudo-para PC step was first assembled sequentially to determine the number of thiophene subunits required to close the macrocycle. ${ }^{12}$ Such chiral macrocycles follow an enantiomerization 
process described first by Kurt Mislow as "Euclidean rubber glove" ${ }^{13-15}$ This means that during the entire enantiomerization process the object remains chiral and achiral arrangements can't be adopted without breaking a bond. The process resembles the transformation of a right-handed rubber glove into a left-handed one by turning it inside out. In the case of $\mathbf{2}$, spontaneous enantiomerization was observed at room temperature due to rotation of the PC subunit around the single bonds integrating it into the oligothiophene macrocycle. $^{12}$

Introduction of bulky rod-like arms with a bis-(para)-pseudo-para orientation ${ }^{16}$ to the PC (figure 1e,f) should gear its free rotation, and thereby allow the separation of the enantiomers. To estimate the dimensions of the target structure 1 , the geometry of one enantiomer, based on the smallest unstrained cyclo[12] thiophene with one thiophene unit replaced by the substituted PC, was optimized by DFT and is depicted as Fig. S1. ${ }^{17,18}$ The calculated distance between the carboxylic oxygen atoms is $\sim 21 \AA$, while the largest distance between two sulfur atoms in the macrocycle is $\sim 13.5 \AA$ (figure S1). For the enantiomerization to occur, one of the para-ethynyl methylbenzoates must pass through the ring of the macrocycle, like a thread through the eye of a needle. We employed a similar strategy in the synthesis of a molecular turnstyle, where a size mismatch of a pushpull rod and a conjugated macrocyclic ring was used to stabilize atropisomers through hindered rotation. ${ }^{19}$

\section{DISCUSSION}

The synthesis (scheme 1) of target macrocycle 1 started from the literature-known dimethyl 7,13-dibromo[2.2] paracyclophane4,16-dicarboxylate and trimethyl (5-(4,4,5,5-tetramethyl-1,3,2-dioxaborolan-2-yl)thiophen-2-yl)silane (3) which were coupled under Suzuki-Miyaura conditions. ${ }^{16,20}$ Conversion of the trimethylsilylgroup of $\mathbf{4}$ to bromine proceeded in high yield but had to be performed in dimethylformamide at $70^{\circ} \mathrm{C}$ since the obtained building block 5 was poorly soluble in other common solvents. Subsequently, 5 was reacted under Pd-PEPPSI ${ }^{\mathrm{TM}}$-IPr catalysis with 3-hexylthiophene-2-boronic acid pinacol ester yielding tetrathiophene 6 . Suitable crystals for single-crystal X-ray analysis of 6 (Fig. S18) were obtained from a dichloromethane (DCM) ethanol mixture. The methyl esters of 6 were then reduced with diisobutylaluminiumhydride (DIBAL-H) and subsequently oxidized with Bobitt's salt to give dialdehyde 7. It was necessary to control the amount of DIBAL-H precisely, because more than four equivalents of the reagent led to complex product mixtures. Halogenation of 7 leads to a poorly soluble intermediate, which was directly reacted with 3 under $\mathrm{Pd}$ PEPPSI $^{\mathrm{TM}}$-IPr catalysis to give hexathiophene 8. Conversion of the TMS groups of $\mathbf{8}$ to the corresponding dibromide yielded $\mathbf{9}$ in excellent yield. Next, building block 12 was assembled from triisopropyl(thiophene-2-ylethynyl)silane, which was converted to pinacolboronic ester 10. Suzuki-Miyaura coupling afforded intermediate

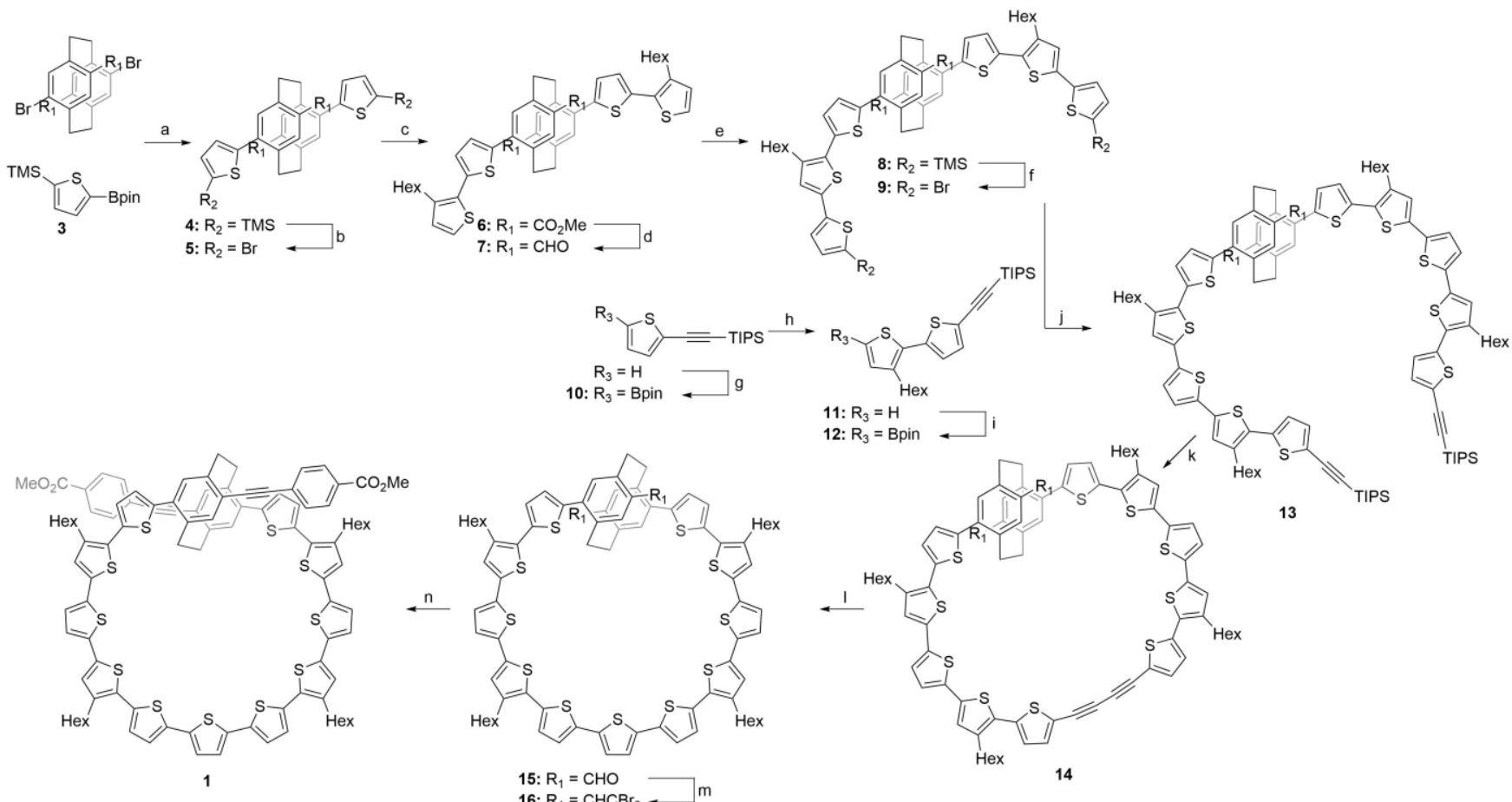

Scheme 1. Synthesis of target compound 1. Reaction conditions: a) 3, $\mathrm{Pd}(\mathrm{dppf}) \mathrm{Cl}_{2}, \mathrm{~K}_{3} \mathrm{PO}_{4}$, toluene $/ \mathrm{H}_{2} \mathrm{O} 10: 1,2 \mathrm{~h}, 110^{\circ} \mathrm{C}, 83 \%$; b) NBS, DMF,

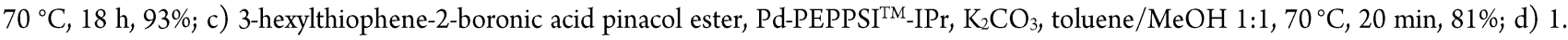
DIBAL-H, THF, $0^{\circ} \mathrm{C}, 3$ h; 2. Bobitt's salt, silica gel, $\mathrm{CH}_{2} \mathrm{Cl}_{2}$, rt, 18 h, 79\%; e) 1. NBS, $\mathrm{CHCl}_{3}$, rt, 18 h; 2. 3, Pd-PEPPSI ${ }^{\mathrm{TM}}-\mathrm{IPr}_{2} \mathrm{~K}_{2} \mathrm{CO}_{3}$, toluene/ $\mathrm{MeOH} \mathrm{1:1,70}{ }^{\circ} \mathrm{C}, 30 \mathrm{~min}, 89 \%$; f) NBS, AcOH/CHCl $1: 1$, rt, 16 h, 99\%; g) n-BuLi, 2-isopropoxy-4,4,5,5-tetramethyl-1,3,2-dioxaborolane, $\mathrm{THF},-78^{\circ} \mathrm{C}$ to rt, $18 \mathrm{~h}, 99 \%$; h) 2-bromo-3-hexylthiophene, Pd-PEPPSI ${ }^{\mathrm{TM}}-\mathrm{IPr}, \mathrm{K}_{2} \mathrm{CO}_{3}$, toluene $/ \mathrm{MeOH} 1: 1,70{ }^{\circ} \mathrm{C}, 20 \mathrm{~min}, 51 \%$; i) n-BuLi, THF, 2-isopropoxy-4,4,5,5-tetramethyl-1,3,2-dioxaborolane, $-78^{\circ} \mathrm{C}$ to rt, $18 \mathrm{~h}, 97 \%$; j) Pd-PEPPSI ${ }^{\mathrm{TM}}$-IPr, $\mathrm{K}_{2} \mathrm{CO}_{3}$, toluene $/ \mathrm{MeOH} 1: 1,70{ }^{\circ} \mathrm{C}, 30 \mathrm{~min}$, $72 \%$; k) 1. TBAF, THFaq, $16 \mathrm{~h} ; 2 . \mathrm{CuCl}, \mathrm{Cu}(\mathrm{OAc})_{2}$, pyridine, $80^{\circ} \mathrm{C}, 40 \mathrm{~h}$ (syringe pump), 85\%; 1 ) $\mathrm{Na}_{2} \mathrm{~S} \cdot 9 \mathrm{H}_{2} \mathrm{O}$, DMF/2-methoxyethanol 1:1, 122 $\left.{ }^{\circ} \mathrm{C}, 2 \mathrm{~h}, 94 \% ; \mathrm{m}\right) \mathrm{CBr}_{4}, \mathrm{PPh}_{3}, \mathrm{CH}_{2} \mathrm{Cl}_{2}, 0^{\circ} \mathrm{C}, 1 \mathrm{~h}, 80 \%$; n) 1. LDA, THF, $0{ }^{\circ} \mathrm{C}$ to rt; 2. methyl-4-iodobenzoate, $\mathrm{Pd}\left(\mathrm{PPh}_{3}\right)_{4}$, CuI, THF, diisopropylamine, $55^{\circ} \mathrm{C}, 2 \mathrm{~h}, 18 \%$. 
11, which was converted to pinacolboronic ester 12. Another $\mathrm{Su}$ zuki-Miyaurareaction of $\mathbf{1 2}$ and $\mathbf{9}$ afforded decathiophene 13, which was first deprotected with tetrabutylammonium fluoride and subjected directly to modified Eglinton coupling conditions. Macrocycle $\mathbf{1 4}$ was obtained in excellent yield of $85 \%$ and was reacted with $\mathrm{Na}_{2} \mathrm{~S}$ to give undecathiophene macrocycle 15 that contains all eleven thiophene units of the target compound $\mathbf{1}$. The rod-like arms were installed by treating 15 with the Corey-Fuchs reaction sequence, ${ }^{[21]}$ converting the dialdehyde into the diacetylene, which was directly subjected to Sonogashira-Hagihara coupling conditions with 4-iodomethylbenzoate to yield the target molecule 1 that was purified by recycling gel permeation chromatography in chloroform after aqueous work-up. Compound $\mathbf{1}$ showed prolonged stability to air and light as a solid. It was characterized by ${ }^{1} \mathrm{H}$, as well as $2 \mathrm{D}$ NMR and UV/Vis spectroscopy, and high-resolution MALDI-TOF spectrometry (see the supporting information). Notably, the ${ }^{1} \mathrm{H}$ NMR spectrum showed two separate resonances of the two methyl esters, indicating that the enantiomerization is slow on the timescale of the NMR experiment. The isotopic pattern recorded in the MALDITOF mass spectrometry experiment matched the elemental composition of $\mathbf{l}\left([\mathrm{M}]^{+} \mathrm{C}_{104} \mathrm{H}_{98} \mathrm{O}_{4} \mathrm{~S}_{11}\right)$.

The enantiomers of 1 were separated by HPLC on a chiral stationary phase (Chiralpak IG, eluent ethylacetate/ $n$-hexane 1:4, $1.0 \mathrm{~mL}$ $\mathrm{min}^{-1}$, column oven: $\mathrm{T}=18^{\circ} \mathrm{C}$ ). The isolated fractions of enantiomers displayed identical MALDI-TOF mass spectra and mirror images of their electronic circular dichroism (ECD) spectra with a set of Cotton effects at 265, 283, 298, 333, 364, 408, 457, and $536 \mathrm{~nm}$ (figure 2a). The Cotton effects are of low intensity compared with e.g. those observed for doubly-helical PC macrocycles of Chujo and coworkers, ${ }^{22,23}$ which probably reflects the high flexibility of the PC unit in the oligothiophene macrocycle. It should be noted that despite all precautions (see experimental section) a partial racemization of the samples could occur during the course of the ECD measurements. The absolute configuration of the enantiomers was revealed by simulation of the ECD spectra with TD-DFT calculations

$$
\text { a) }
$$

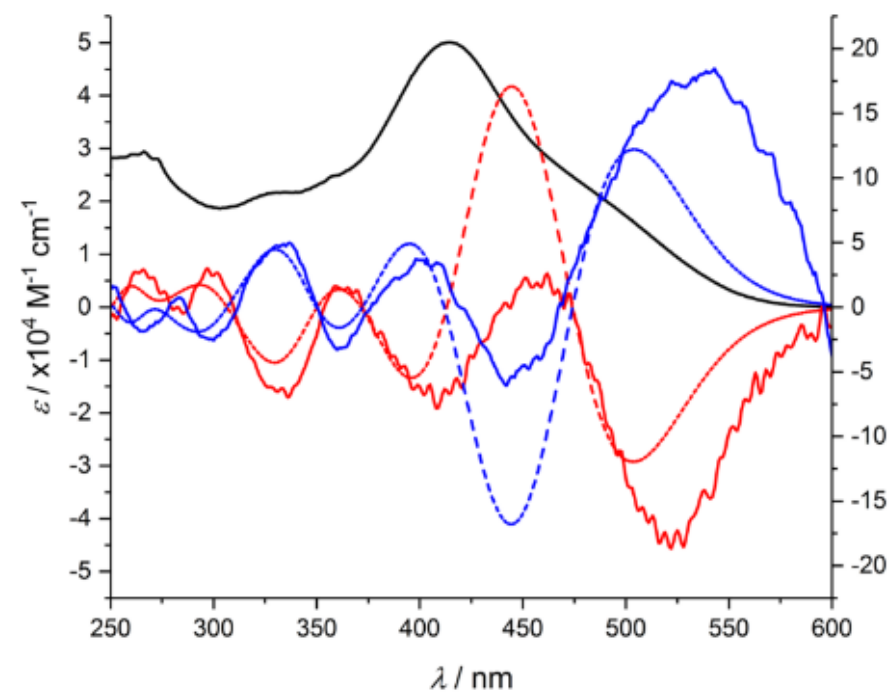

(see supporting information (SI)) that allowed to assign the (M)and the $(P)$-configuration to the first and the second eluted fraction, respectively, in the chiral HPLC separation (figure $2 b$ ). ${ }^{24,25}$

Reinjection of an initially pure enantiomer of $\mathbf{1}$ stored at room temperature (rt) for several minutes already displayed the appearance of the other enantiomer, pointing at racemization at $r t$ in spite of the length of the rod-like arms which were mounted to hinder the $\mathrm{PC}$ rotation. Thus, the enantiomerization behavior of $\mathbf{1}$ was investigated by means of dynamic variable temperature (VT) HPLC on a chiral stationary phase. The elution profiles of $\mathbf{l}$ in the temperature range from 293 to $328 \mathrm{~K}$ in steps of $5 \mathrm{~K}$ (eluent ethylacetate/n-hexane 2:3) was recorded and analyzed (see SI) with the DCXplorer ${ }^{26}$ and the dHPLC Y $2 \mathrm{~K}^{27,28}$ software packages. We found the half-life of enantiomerization is only $t_{1 / 2}{ }^{298 \mathrm{~K}}=15.9 \pm 0.5 \mathrm{~min}$ at $298 \mathrm{~K}$ with the activation free energy calculated as $\Delta G^{\ddagger} 298 \mathrm{~K} \approx 91 \mathrm{~kJ} \mathrm{~mol}^{-1}$ by both programs. The VT HPLC experiments further allowed for constructing an Eyring plot to dissect the free energy barrier into the corresponding enthalpy $\left(\Delta H_{\mathrm{e}}^{\dagger} \approx 37-42 \mathrm{~kJ} \mathrm{~mol}^{-1}\right)$, and entropy $\left(\Delta S_{\mathrm{e}}^{\dagger}<\right.$ $\left.-160 \mathrm{~J} \mathrm{~mol}^{-1} \mathrm{~K}^{-1}\right)$ contributions. The values determined for $\Delta H_{e}^{\ddagger}$ and $\Delta S_{\mathrm{e}}{ }^{\dagger}$ are striking because they suggest that the PC rotation is to a large extent controlled by a vast entropic penalty. Considering the size of the macrocycle 1 and the length and the stiffness of the methyl 4-ethynylbenzoate arms, the $\Delta H_{e}^{\ddagger}$ values appear to be intriguingly

b)

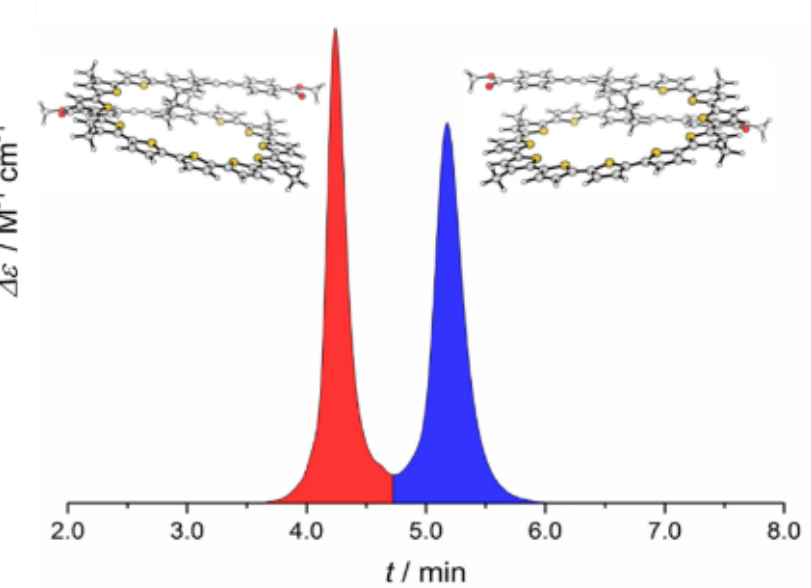

Figure 2. a) UV/Vis spectrum (black line) of a DCM solution of 1, experimental (solid lines; recorded at $10^{\circ} \mathrm{C}$ in $n$-hexane/EtOAc $4: 1$ ) and calculated (dashed lines) ECD spectra of $(M)-1$ (red) and (P)-1 (blue). b) Representative elution profile from the VT HPLC experiment (298 K column temperature, detected at $409 \mathrm{~nm}):(M)-1$ (red area) and $(P)-1$ (blue area). The model shows the absolute configuration (the hexyl chains are replaced with methyl groups for clarity). 

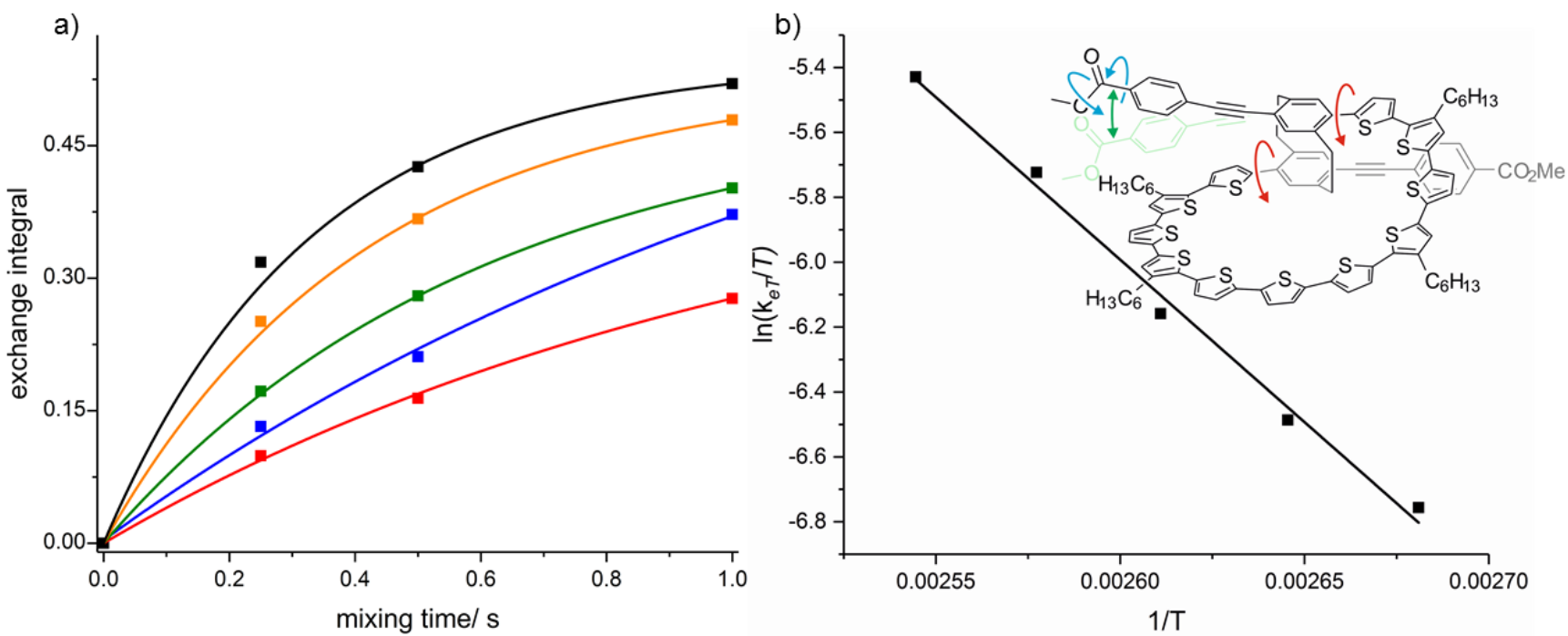

Figure 3. a) Exchange integral against mixing time in the VT EXSY experiment (red: $373 \mathrm{~K}$, blue: $378 \mathrm{~K}$, green: $383 \mathrm{~K}$, orange: $388 \mathrm{~K}$, black: 393 K).b) Eyring plot from data obtained from VT EXSY experiment. Linear fit to the data provides the enthalpy $\left(\Delta \mathrm{H}_{e}^{\ddagger}=83 \mathrm{~kJ} \mathrm{~mol}^{-1}\right)$ and the entropy $\left(\Delta S_{\mathrm{e}}^{\dagger}=-31 \mathrm{~J} \mathrm{~mol}^{-1} \mathrm{~K}^{-1}\right)$ for the racemization process. The arrows indicate specific geometric changes obtained from calculations (red: PC rotation, green: arm bending, blue: methoxy or carboxylate rotations).

low. The preexponential factor $\log (A) \approx 5\left(E_{\mathrm{a}} \approx 45 \mathrm{~kJ} \mathrm{~mol}^{-1}\right)$ for enantiomerization of $\mathbf{1}$, which is a unimolecular process, obtained from Arrhenius analysis suggests that the temperature dependence of the rate constant obtained from the elution profile analysis may be inaccurate. Indeed, it has been described previously that the elution profile analysis from dynamic VT HPLC experiments provides accurate values of activation free energy but tends to overestimate the entropy contribution when compared to alternative methods like dynamic ECD, VT NMR spectroscopies, or quantum-chemical calculations. ${ }^{29}$ Possibly, the heterogenous nature of the HPLC experiment, where equilibria of the analyte with the chiral stationary phase are involved, results in enthalpy-entropy compensation affecting the individual activation parameters. As alternative access to these values, a 1,1,2,2-tetrachloroethane- $d_{2}$ solution of racemic 1 was studied by VT 2D exchange spectroscopy (EXSY) in a temperature range of 373-393 $\mathrm{K}$ in steps of $5 \mathrm{~K}$ (see figure 3a and SI) to determine the rate constants. Indeed, in agreement with our expectation, an enthalpy dominated racemization process was monitored with $\Delta H_{\mathrm{e}}^{\ddagger}=83 \mathrm{~kJ} \mathrm{~mol}^{-1}$ and $\Delta S_{\mathrm{e}}^{\ddagger}=-31 \mathrm{~J} \mathrm{~mol}^{-1} \mathrm{~K}^{-1}$ obtained from the corresponding Eyringplot (figure 3b). The activation free energy at 298 $\mathrm{K}$ however, was with $\Delta G^{\ddagger}{ }_{298 \mathrm{~K}}=92.4 \mathrm{~kJ} \mathrm{~mol}^{-1}$ comparable with the value obtained by the dynamic VT HPLC experiments.

We performed quantum-chemical calculations to get a deeper insight into the enantiomerization mechanism. The size and the flexible nature of macrocycle $\mathbf{1}^{30}$ coerced us to use a combination of semiempirical and DFT calculations (see SI for detailed description) at the PM6 and B3LYP level of theory, respectively. First, we performed a PM6 relaxed potential energy surface scan (PES scan) for 1 along the dihedral angle $\alpha$ that is defined as the angle between the planes of the PC benzene rings and those of the two adjacent thiophene units to mimic the PC rotation. ${ }^{31}$ Note that clockwise and anticlockwise $\mathrm{PC}$ rotations are not equivalent because $\mathbf{1}$ is chiral. In any case, we discovered few possible enantiomerization pathways that all require (i) a considerable turn of $\mathrm{PC}$ from its energy minimum geometry, (ii) bending of the para-ethynyl methylbenzoate arm, and, unexpectedly, (iii) a rotation of either (a) the methyl carboxylate by $\sim 180^{\circ}$ or (b) the methoxy group from its more stable $s$-cis to $s$-trans conformation. The latter processes (iii-a,b) decrease the steric repulsion between the methyl carboxylate and the thiophene macrocycle and are inevitable to complete the full PC turn required for enantiomerization. All our attempts to find a transition state for the enantiomerization of $(M)$ - or $(P)$ - $\mathbf{1}$ failed. We found that the energy range of the region of the PM6 PES where we observed the arm to successfully thread through the macrocycle during the constrained optimization was $\sim 94-103 \mathrm{~kJ} \mathrm{~mol}^{-1}$ (figures S16 and S17). This energy range represents, neglecting the heat capacities, the upper boundary of the activation enthalpy, and it is in far better agreement with the VT EXSY experimental data than with that obtained from the VT HPLC. Additional evidence can be obtained by considering the energy cost of each of the processes (i-iii) separately assuming their additivity. This allowed us to use the higher-level B3LYP calculations (see SI for details) to obtain the PC rotation profile (figure S12) for the parent PC macrocycle 2a (figure 1d). Again, an identical dihedral angle $\alpha$ for the two thiophene units was assumed. The calculated activation energy of $\sim 35 \mathrm{~kJ} \mathrm{~mol}^{-1}$ (at $0 \mathrm{~K}$ ) for the PC rotation is in very good agreement with the VT NMR activation free energy determined for $\mathbf{2 b}\left(\Delta G^{\dagger}{ }_{298 \mathrm{~K}}=38 \mathrm{~kJ} \mathrm{~mol}^{-1}\right){ }^{12}$

Constraining the valence and dihedral angles of the bent acetylene unit in the para-ethynyl methylbenzoate arm from the PM6 PES scan while relaxing all other internal coordinates provided (figure S13b) a penalty of $\sim 26 \mathrm{~kJ} \mathrm{~mol}^{-1}$ for process (ii). Finally, rotation (iiia) and (iii-b) in para-ethynyl methylbenzoate was computed ${ }^{32-34}$ to require an activation energy of $\sim 21$ and $\sim 28 \mathrm{~kJ} \mathrm{~mol}^{-1}$, respectively. Addition of the individual contributions ( $\mathrm{i}$-iii) provides a semiquantitative estimate for the enantiomerization enthalpy of $\sim 82-89 \mathrm{~kJ}$ $\mathrm{mol}^{-1}$ in very good agreement with our VT EXSY experiments data, suggesting that the enantiomerization of $\mathbf{1}$ is predominantly controlled by enthalpy.

\section{CONCLUSION}


In summary, we designed and synthesized a helically chiral oligothiophene macrocycle, chirality of which emerges from the incorporated [2.2]paracyclophane. The fast enantiomerization is prevented by employing bulky rod-like arms on the central [2.2]paracyclophane unit that allowed to resolve and characterize the chiroptical properties of individual enantiomers by ECD spectroscopy and DFT calculations. The dynamic variable temperature HPLC and 2D exchange spectroscopy permitted to obtain the activation free energy for the enantiomerization process, and the latter method the reliable values of activation enthalpy and entropy further supported by quantum-chemical calculations. Our calculations also revealed the individual internal rotations and structural deformations necessary for the enantiomerization of the helically chiral macrocycle to occur and confirmed thereby also that it follows Mislow's "Euclidian rubber glove" mechanism.

\section{EXPERIMENTAL SECTION}

Synthesis and Characterization. Experimental procedures and characterization data for all new compounds described in this work are compiled in the supporting information. All commercially available compounds were purchased from Sigma-Aldrich, Acros, Apollo Scientific, Alfa Aesar and Fluorochem and used without further purification. All reactions with reagents that are easily oxidized or hydrolyzed were performed under Argon using Schlenk techniques with anhydrous solvents in glassware, which was dried prior to use. The NMR experiments were performed at 400,500 or $600 \mathrm{MHz}$ proton frequencies. The instruments were equipped with a directobserve $5 \mathrm{~mm} \mathrm{BBFO}$ smart probe ( 400 and $600 \mathrm{MHz}$ ), an indirectdetection $5 \mathrm{~mm}$ BBI probe $(500 \mathrm{MHz})$, or a five-channel cryogenic $5 \mathrm{~mm}$ QCI probe $(600 \mathrm{MHz})$. All probes were equipped with actively shielded z-gradients $(10 \mathrm{~A})$. The chemical shifts are reported in ppm relative to tetramethylsilane or referenced to residual solvent peak and the $\mathrm{J}$ values are given in $\mathrm{Hz}( \pm 0.1 \mathrm{~Hz})$. The HPLC separation of the enantiomers of $\mathbf{1}$ was performed on an HPLC instrument equipped with a diode array UV-Vis detector $(\lambda=200-600 \mathrm{~nm})$. The used column for separation on chiral stationary phase was a Chiralpak IG, $5 \mu \mathrm{m}, 4.6 \times 250 \mathrm{~mm}$, Daicel Chemical Industries Ltd. The samples for CD spectra were eluted in $n$-hexane/EtOAc 4:1, the VTHPLC studies were performed in $n$-hexane/EtOAc 2:1, flow rate 1.0 $\mathrm{mL} \mathrm{min}{ }^{-1}$. The CD spectra were measured in a precooled $1 \mathrm{~cm}$ quartz glass cuvette directly after chiral HPLC at $283 \mathrm{~K}$. All EXSY NMR experiments were performed on a Bruker Avance III NMR spectrometer operating at $600.13 \mathrm{MHz}$ proton frequency. The instrument was equipped with an indirect 5-mm BBI probe with z-gradient. The experiments were performed between 373 and $393 \mathrm{~K}$ and the temperature was calibrated using a glycerol standard showing accuracy within $+/-0.2 \mathrm{~K}$. Temperature equilibration was achieved after $20 \mathrm{~min}$ and only then the EXSY experiments were performed. The mixing times were set to 1000,500 or $250 \mathrm{~ms}$ and the total experiment varied between 68 and $94 \mathrm{~min}$. Manual integration of the EXSY cross peaks, normalized with the diagonal peaks yielded the rate constants which were determined applying the initial rate approximation. Eyring analysis of these rates yielded the enthalpic and entropic contributions to the activation energy.

DFT Calculations. The Gaussian 09 (release D01) program suite was used in all calculations. ${ }^{35}$ The potential energy surface scans were performed at either semi-empirical PM6 or B3LYP/6-31G(d) level of theories. The full gas phase geometry optimizations were done with B3LYP functional and 6-31G(d) basis set, and the character of the stationary point reached was confirmed by a subsequent frequency calculation. The zero-point vibrational energy correction obtained this way was used unscaled. The electronic circular dichroism spectra were obtained at CAM-B3LYP/cc-pVDZ level of theory calculating the first 75 electronic transitions. The velocity form of the rotatory strength was used. Empirical vibrational broadening for the electronic transitions was assumed $(0.16 \mathrm{eV})$ and the position of the absorption bands was empirically shifted by $+20 \mathrm{~nm}$ to match the energy of the first electronic transition. The ECD spectra were simulated in SpecDis (v1.71). ${ }^{36}$

\section{ASSOCIATED CONTENT}

Synthetic procedures and analytical data for all new compounds, HPLC, $\mathrm{CD}$, crystallographic data, results ofDFT calculations, ${ }^{1} \mathrm{H}$ and ${ }^{13} \mathrm{C}-\mathrm{NMR}$ and HRMS spectra are supplied as Supporting Information. This material is available free of charge via the Internet at http://pubs.acs.org."

\section{AUTHOR INFORMATION}

\section{Corresponding Author \\ *marcel.mayor@unibas.ch}

\section{Author Contributions}

The manuscript was written through contributions of all authors. All authors have given approval to the final version of the manuscript.

\section{Funding Sources}

We acknowledge financial support by the European FP7-ITN MOLESCO (project no. 606728), the Swiss National Science Foundation (SNF, grant no. 200020-178808 (M.M.) and PZ00P2_174175 (T.S.)), and the Swiss Nanoscience Institute (SNI). M.M. acknowledges support by the 111 project (90002-18011002).

\section{ACKNOWLEDGMENT}

Dedicated to Prof. Heinz Langhals on the occasion of his 70th birthday. We would like to thank the Mass Spectrometry team in the Molecular and Biomolecular Analysis service at ETH Zurich for their expert help in providing high resolution measurements of the synthesized compounds. We thank Marco Pierini (University of Rome, Italy) for dHPLC Y2K calculations and Oliver Trapp (University of Munich, Germany) for providing DCXplorer. The SciCore cluster at the University of Basel is acknowledged for the computational resources.

\section{REFERENCES}

(1) Rickhaus, M.; Mayor, M.; Juríček, M. Strain-Induced Helical Chirality in Polyaromatic Systems. Chem Soc Rev 2016, 45, 1542-1556.

(2) Berezhnaia, V.; Roy, M.; Vanthuyne, N.; Villa, M.; Naubron, J.V.; Rodriguez, J.; Coquerel, Y.; Gingras, M. Chiral Nanographene Propeller Embedding Six Enantiomerically Stable [5]Helicene Units. J. Am. Chem. Soc. 2017, 139, 18508-18511.

(3) Fujikawa, T.; Preda, D. V.; Segawa, Y.; Itami, K.; Scott, L. T. Corannulene-Helicene Hybrids: Chiral $\pi$-Systems Comprising Both Bowl and Helical Motifs. Org. Lett.2016, 18, 3992-3995.

(4) Schuster, N.J.; Paley, D. W.; Jockusch, S.; Ng, F.; Steigerwald, M. L.; Nuckolls, C. Electron Delocalization in Perylene Diimide Helicenes. Angew. Chem. Int. Ed. 2016, 55, 13519-13523. 
(5) Gohler, B.; Hamelbeck, V.; Markus, T. Z.; Kettner, M.; Hanne, G. F.; Vager, Z.; Naaman, R.; Zacharias, H. Spin Selectivity in Electron Transmission Through Self-Assembled Monolayers of Double-Stranded DNA. Science 2011, 331, 894-897.

(6) Iyoda, M.; Yamakawa, J.; Rahman, M. J. Conjugated Macrocycles: Concepts and Applications. Angew. Chem. Int. Ed. 2011, 50, $10522-$ 10553.

(7) Brown, C. J.; Farthing, A. C. Preparation and Structure of Di-pXylylene. Nature 1949, 164, 915-916.

(8) Cram, D. J.; Steinberg, H. Macro Rings. I. Preparation and Spectra of the Paracyclophanes. J. Am. Chem. Soc. 1951, 73, 5691-5704.

(9) Hinrichs, H.; Boydston, A. J.; Jones, P. G.; Hess, K.; Herges, R.; Haley, M. M.; Hopf, H. Phane Properties of [2.2]Paracyclophane/Dehydrobenzoannulene Hybrids. Chem. - Eur. J. 2006, 12, 7103-7115.

(10) Morisaki, Y.; Gon, M.; Sasamori, T.; Tokitoh, N.; Chujo, Y. Planar Chiral Tetrasubstituted [2.2]Paracyclophane: Optical Resolution and Functionalization. J. Am. Chem. Soc. 2014, 136, 3350-3353.

(11) Stefani, D.; Weiland, K. J.; Skripnik, M.; Hsu, C.; Perrin, M. L.; Mayor, M.; Pauly, F.; van der Zant, H. S. J. Large Conductance Variations in a Mechanosensitive Single-Molecule Junction. Nano Lett. 2018, 18, 59815988.

(12) Weiland, K. J.; Münch, N.; Gschwind, W.; Häussinger, D.; Mayor, M. A Chiral Macrocyclic Oligothiophene with Broken Conjugation - Rapid Racemization through Internal Rotation. Helv. Chim. Acta 2018, 101, e1800205.

(13) Flapan, E. When Topology Meets Chemistry: A Topological Look at Molecular Chirality; Outlooks; Cambridge University Press ; Mathematical Association of America: Cambridge ; New York : Washington, DC, 2000.

(14) Chambron, J.-C.; Sauvage, J.-P.; Mislow, K.; De Cian, A.; Fischer, J. A [2]Catenane and a [2]Rotaxane as Prototypes of Topological and Euclidean Molecular "Rubber Gloves." Chem. - Eur. J. 2001, 7, 40854096.

(15) Mislow, K. Limitations of the Symmetry Criteria for Optical Inactivity and Resolvability Science 1954, 120, 232-233.

(16) Vorontsova, N. V.; Rozenberg, V. I.; Sergeeva, E. V.; Vorontsov, E. V.; Starikova, Z. A.; Lyssenko, K. A.; Hopf, H. Symmetrically Tetrasubstituted [2.2]Paracyclophanes: Their Systematization and Regioselective Synthesis of Several Types of Bis-Bifunctional Derivatives by Double Electrophilic Substitution. Chem. - Eur. J. 2008, 14, 4600-4617.

(17) Krömer, J.; Rios-Carreras, I.; Fuhrmann, G.; Musch, C.; Wunderlin, M.; Debaerdemaeker, T.; Mena-Osteritz, E.; Bäuerle, P. Synthesis of the First Fullya-Conjugated Macrocyclic Oligothiophenes: Cyclo[n]Thiophenes with Tunable Cavities in the Nanometer Regime. Angew. Chem. 2000, 39, 3481-3486.

(18) Mishra, A.; Ma, C.-Q.; Bäuerle, P. Functional Oligothiophenes: Molecular Design for Multidimensional Nanoarchitectures and Their Applications. Chem. Rev. 2009, 109, 1141-1276.

(19) Le Pleux, L.; Kapatsina, E.; Hildesheim, J.; Häussinger, D.; Mayor, M. A Molecular Turnstile as an E-Field-Triggered Single-Molecule Switch: Concept and Synthesis: A Molecular Turnstile as an $E$-Field-Triggered Single-Molecule Switch: Concept and Synthesis. Eur. J. Org. Chem. 2017, 2017, 3165-3178.

(20) Thirion, D.; Poriel, C.; Métivier, R.; Rault-Berthelot, J.; Barrière, F.; Jeannin, O. Violet-to-Blue Tunable Emission of Aryl-Substituted Dispirofluorene-Indenofluorene Isomers by Conformationally-Controllable Intramolecular Excimer Formation. Chem. - Eur. J. 2011, 17, 1027210287.

(21) Corey, E. J.; Fuchs, P. L. A Synthetic Method for Formyl -> Ethynyl Conversion. Tetrahedron Lett. 1972, 13, 3769-3772.

(22) Morisaki, Y.; Kawakami, N.; Shibata, S.; Chujo, Y. ThroughSpace Conjugated Molecular Wire Comprising Three $\pi$-Electron Systems. Chem. - Asian J.2014, 9, 2891-2895.
(23) Gon, M.; Morisaki, Y.; Sawada, R.; Chujo, Y. Synthesis of Optically Active, X-Shaped, Conjugated Compounds and Dendrimers Based on Planar Chiral [2.2]Paracyclophane, Leading to Highly Emissive Circularly Polarized Luminescence. Chem. - Eur. J.2016, 22, 2291-2298.

(24) Yanai, T.; Tew, D. P.; Handy, N. C. A New Hybrid ExchangeCorrelation Functional Using the Coulomb-Attenuating Method (CAMB3LYP). Chem. Phys. Lett. 2004, 393, 51-57.

(25) Bruhn, T.; Schaumlöffel, A.; Hemberger, Y.; Bringmann, G. SpecDis: Quantifying the Comparison of Calculated and Experimental Electronic Circular Dichroism Spectra: UV AND ECD EVALUATION WITH SPECDIS. Chirality 2013, 25, 243-249.

(26) Trapp, O. A Novel Software Tool for High Throughput Measurements of Interconversion Barriers: DCXplorert. J. Chromatogr. B2008, $875,42-47$.

(27) D’Acquarica, I.; Gasparrini, F.; Pierini, M.; Villani, C.; Zappia, G. Dynamic HPLC on Chiral Stationary Phases: A Powerful Tool for the Investigation of Stereomutation Processes. J. Sep. Sci. 2006, 29, 1508-1516.

(28) Rizzo, S.; Benincori, T.; Bonometti, V.; Cirilli, R.; Mussini, P. R.; Pierini, M.; Pilati, T.; Sannicolò, F. Steric and Electronic Effects on the Configurational Stability of Residual Chiral Phosphorus-Centered Three-Bladed Propellers: Tris-Aryl Phosphanes. Chem. - Eur. J. 2013, 19, 182-194.

(29) Bihlmeier, A.; Rotzler, J.; Rickhaus, M.; Mayor, M.; Klopper, W. Activation Enthalpies and Entropies of the Atropisomerization of Substituted Butyl-Bridged Biphenyls. Phys. Chem. Chem. Phys. 2015, 17, 1116511173.

(30) Even with the solubilizing hexyl chains reduced to methyl groups or hydrogen atoms.

(31) Although the dihedral angles between the plane of each of the thiophene units and the plane of the top or the bottom benzene ring of the $\mathrm{PC}$, respectively, are not necessarily identical, a single variable $\alpha$ had to be used to simulate the PC rotation to avoid rotation of the thiophene unit when a single dihedral angle was constrained, while the other relaxed in the optimization.

(32) The B3LYP PES calculations for both rotations overestimate the activation energy of each process. Therefore, both were corrected by the difference of the activation barrier obtained from the PES calculated for methyl benzoate and methyl formate at the same level of theory and the experimental values reported in the literature (refs. 33 and 34).

(33) Bailey, J.; North, A. M. Ultrasonic Relaxation in Some Alkyl Esters. Trans. Faraday Soc. 1968, 64, 1499.

(34) Pawar, D. M.; Wilson, K. K.; Noe, E. A. Barrier to Rotation about the Phenyl-Carbonyl Carbon Bond of Methyl Benzoate by Dynamic NMR Spectroscopy and Ab Initio Molecular Orbital Calculations. J. Org. Chem. 2000, 65, 1552-1553.

(35) Frisch, M. J.; Trucks, G. W.; Schlegel, H. B.; Scuseria, G. E.; Robb, M. A.; Cheeseman, J. R.; Scalmani, G.; Barone, V.; Petersson, G. A.; Nakatsuji, H.; Li, X.; Caricato, M.; Marenich, A. V.; Bloino, J.; Janesko, B. G.; Gomperts, R.; Mennucci, B.; Hratchian, H. P.; Ortiz, J. V.; Izmaylov, A. F.; Sonnenberg, J. L.; Williams-Young, D.; Ding, F.; Lipparini, F.; Egidi, F.; Goings, J.; Peng, B.; Petrone, A.; Henderson, T.; Ranasinghe, D.; Zakrzewski, V. G.; Gao, J.; Rega, N.; Zheng, G.; Liang, W.; Hada, M.; Ehara, M.; Toyota, K.; Fukuda, R.; Hasegawa, J.; Ishida, M.; Nakajima, T.; Honda, Y.; Kitao, O.; Nakai, H.; Vreven, T.; Throssell, K.; Montgomery, J. A., Jr.; Peralta, J. E.; Ogliaro, F.; Bearpark, M. J.; Heyd, J.J.; Brothers, E. N.; Kudin, K. N.; Staroverov, V. N.; Keith, T. A.; Kobayashi, R.; Normand, J.; Raghavachari, K.; Rendell, A. P.; Burant, J. C.; Iyengar, S. S.; Tomasi, J.; Cossi, M.; Millam, J. M.; Klene, M.; Adamo, C.; Cammi, R.; Ochterski,J. W.; Martin, R. L.; Morokuma, K.; Farkas, O.; Foresman, J. B.; Fox, D. J. Gaussian 09, Revision D.01, Gaussian, Inc., Wallingford CT, 2009.

(36) Bruhn, T.; Schaumlöffel, A.; Hemberger, Y; Bringmann, G. SpecDis: Quantifying the Comparison of Calculated and Experimental Electronic Circular Dichroism Spectra. Chirality 2013, 25, 243-249. 


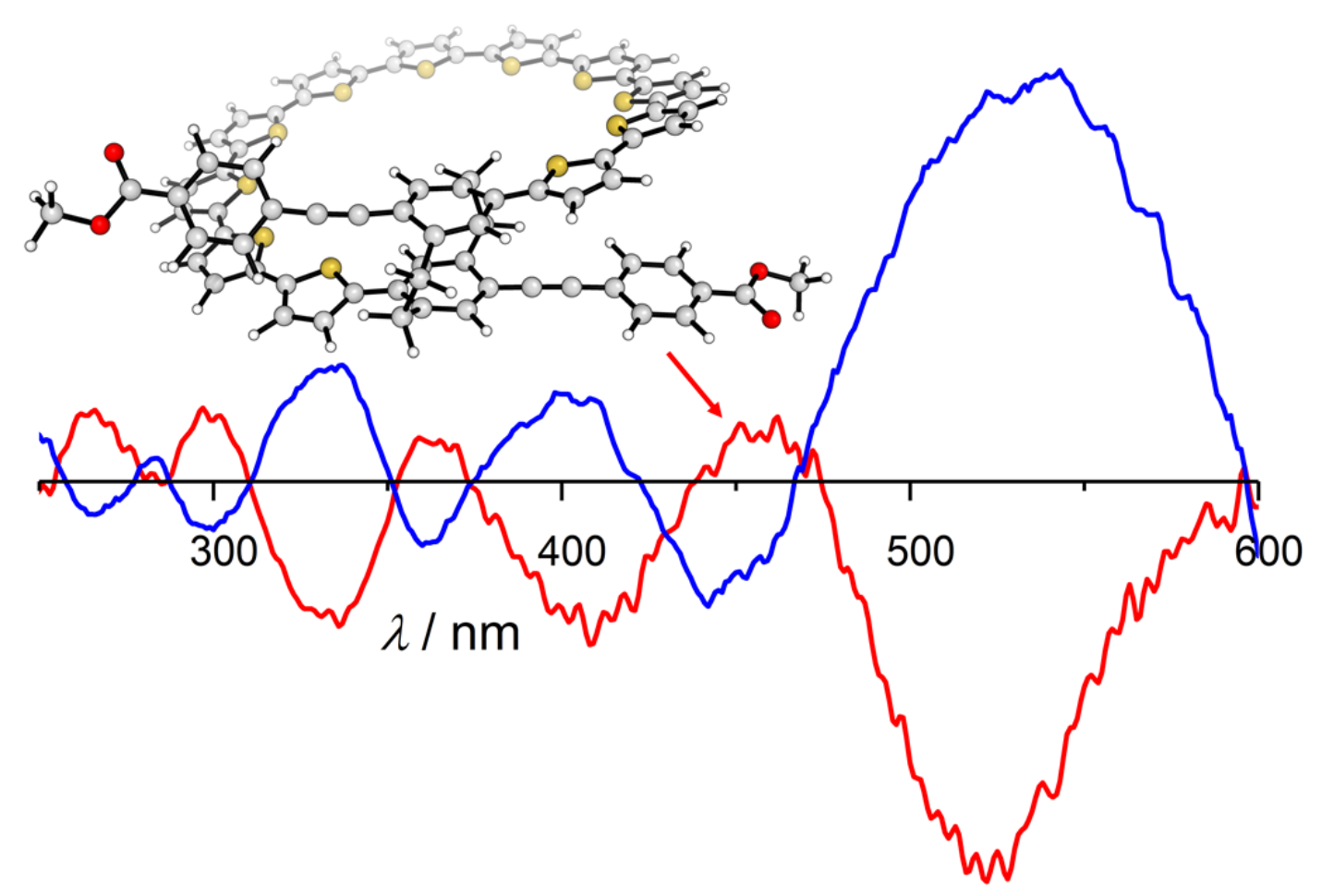

